\title{
Motion Estimation via a Zoom Camera
}

\author{
Lili Ma, Chengyu Cao, Amanda Young, Naira Hovakimyan*
}

\begin{abstract}
This paper discusses the motion estimation problem of a rigid-body using a zoom camera via observations of a single feature point. Both the rotational and translational parameters are assumed to be unknown constants. Estimation of the unknown parameters is obtained in two steps. In the first step, the camera's focal length is fixed and the rotational parameters can be estimated. In the second step, the camera's focal length is changing as a time-varying signal. In our current design, the focal length performs a sinusoidal motion. The unknown depth, along with the translational parameters, can be estimated. Simulation results are presented that demonstrate successful motion estimation.
\end{abstract}

Key Words: Motion estimation, depth from zooming.

\section{Introduction}

The problem of 3D motion estimation has been a central topic in development of vision-based navigation and control algorithms. Solutions to this problem can perceivably affect applications such as vision-based target tracking, coordination, navigation and obstacle avoidance. Various solutions to this problem have been proposed, such as nonlinear optimization techniques ${ }^{1-3}$, linear least-square methods ${ }^{4}$, and extended Kalman filter ${ }^{5,6}$, in addition to a class of nonlinear observers that arise from a control view point. These nonlinear observers, typically named as perspective nonlinear observers, study the perspective dynamic systems that result from the perspective projection. In general, a perspective dynamic system is a linear system whose output is observed up to a homogeneous line. It is due to this perspective projection that depth ambiguity occurs.

The problem of 3D motion estimation via perspective nonlinear observers has evolved through two stages in its development. First, range identification was addressed, where all the motion parameters of the moving object have been assumed to be known. The objective was to estimate the depth, or, otherwise saying, the range. A variety of nonlinear observers have been proposed, including the identifier-based observer (IBO) ${ }^{7}$, the sliding mode-based observer ${ }^{8}$, the Lyapunovbased method ${ }^{9}$, the linear approximation-based observer ${ }^{10}$, and the reduced-order observer ${ }^{11}$, to name a few. Next, 3D motion estimation was considered that estimates both the unknown depth/position and all (or some) of the unknown motion parameters by employing stereo vision ${ }^{12}$, running multiple cameras simultaneously ${ }^{13}$, integrating vision with range sensors ${ }^{14}$, active vision (moving the camera on a rod) ${ }^{15}$, or by modifying the behavior of the follower on which the camera

*Lili Ma and Amanda Young are with the Dept. of Aerospace and Ocean Engineering, Virginia Tech, Blacksburg, VA 24061. Emails: \{lma05,ayoung83@vt.edu\}@vt.edu. Chengyu Cao is with the Dept. of Mechanical Engineering, University of Connecticut, Storrs, CT 06269. Email: ccao@engr.uconn.edu. Naira Hovakimyan is with the Dept. of Mechanical Science and Engineering, University of Illinois at Urbana-Champaign, Urbana, IL 61801. Email: nhovakim@illinois.edu 
is installed to provide persistent excitation to recover the target's information ${ }^{16}$. Alternatively, in this paper, we present a solution to the $3 \mathrm{D}$ motion estimation problem by employing a single zoom camera.

Inferring 3D information from a set of images taken by a zoom camera has been discussed in Refs. 17-22. It was reported in Refs. 18,20,22 that the pinhole projection model is inadequate to model the zooming effect, and more complicated models, such as thin-lens and thick-lens models, have to be used. In the aforementioned depth recovery results, the distance between the camera and the scene (target) is assumed to be fixed. The problem addressed aims at recovering the relative distance by taking several (at least two) shots of the scene/target with different zooming scales. The fundamental method is based on either direct computation using two views ${ }^{17,20,22}$, or leastsquare minimization using multiple views ${ }^{21}$. In this paper, we exploit a recursive approach where estimation of the 3D information is updated upon acquiring each new image. More specifically, we discuss the 3D motion estimation problem of a rigid body, by observing one single feature point on the object and assuming that the object is moving according to a linear rigid-body motion. We appeal to nonlinear observer(s) and design of a time-varying motion of the camera's adjustable focal length to recover all the unknown motion parameters and the (absolute) 3D position of the target.

We show that 3D motion estimation of a rigid-body motion can be achieved via a zoom camera through two separate steps. In the first step, the zoom camera is used as a fixed focal-length camera. The three rotational parameters of the rigid-body motion can be estimated in this step, similar to the method presented in Ref. 12. The zoom camera's focal length performs a time-varying motion in the second step to enable estimation of the unknown depth and the translational parameters by providing persistent excitation, and thus eliminating the need for additional information or extra sensors. Therefore, by employing the use of an intrinsic parameter unique to the mechanical active vision system, i.e., the adjustable focal length (also known as zoom), depth recovery and motion estimation of a rigid-body motion can be achieved without modifying the vehicles' behavior or utilizing more sensors. Apparently, utilizing a zoom camera avoids the requirement of an additional camera as in the stereo vision setup. The image processing task can therefore be simplified.

Our proposed method is feasible due to two factors. First, it is assumed that the motion parameters are unknown constants. This renders the resulting perspective dynamic systems into linearly parameterized structure so that existing perspective nonlinear observers can be applied. These perspective nonlinear observers include those proposed in Refs. 7-9, 11, 12, 23-27, to name but a few. In this paper, the IBO described in Refs. 7, 28, 29 is used for state and parameter estimation. Next, the depth ambiguity is resolved by implementing adjustable focal length.

The work described in this paper stems from a project on vision-based target tracking of a ground vehicle for a small unmanned aerial vehicle equipped with a gimbaled Pan-Tilt-Zoom camera ${ }^{30-33}$. This paper is organized as follows. Section II briefly reviews several projection models for a zoom camera where the thin-lens model is utilized in this paper for the purpose of 3D motion estimation. Detailed procedures of our proposed method are described in Sec. III. Simulation results are given in Sec. IV, Finally, Section V concludes the paper.

\section{Projection Models for a Zoom Camera}

The degrees of freedom in active vision systems include both extrinsic parameters (similar to eye movements) and intrinsic parameters (eye configuration). We are interested in exploring the use 
of the adjustable focal length (also known as zoom), an intrinsic parameter unique to mechanical active vision. To explain the optical phenomenon occurring during a zoom-lens focal-length change, it has been observed that the simplest pinhole model is inadequate and more complicated models must be considered $18,20,22$.

There are four camera projection models currently in use ${ }^{17,20,34}$ :

1. Pinhole Model: The pin-hole model assumes that all light rays coming from the object focus through a single point (the focal point) onto the image plane. This geometrically simple model is widely used in computer vision. However, it is only accurate if the focal length, i.e., the distance between the image plane and the focal point, is negligible compared to the object distance (e.g., in aerial photography) ${ }^{20}$.

2. Thin-Lens Model: The thin-lens model assumes an infinitely thin lens, modelled by a plane. Light rays from the object hitting the plane at a particular angle continue after leaving the plane at a modified angle ${ }^{17}$.

3. Thick-Lens Model: The thick-lens model represents a lens by two planes called the principle planes. Light rays entering one plane at a specific angle travel parallel to the optical axis through to the second plane, from which they exit at another angle (similar to the thin-lens model). Ignoring aperture effects, this model is equivalent to the pinhole model with the addition of a virtual axial motion, and it is sufficient for modeling zoom lenses, even at close range 20,34 .

4. Geometric-Lens Model: The geometric-lens model is based on a complete geometric description of all of the glass elements of the lens. It is the most detailed and accurate of the models, and can be used to design lenses and to model effects such as optical abberations, lens flare, etc. However, this model is extremely complicated to implement ${ }^{20}$.

Let $[X, Y, Z]^{\top}$ be the coordinates of a point in the camera frame. Projections on the image plane when using the thin-lens and the thick-lens models are summarized in the following:

1. Thin-Lens Model ${ }^{17}$ :

$$
\left[\begin{array}{l}
x \\
y
\end{array}\right]=\frac{-f}{Z-f}\left[\begin{array}{l}
X \\
Y
\end{array}\right],
$$

where $f$ denotes the camera's focal length.

2. Thick-Lens Model ${ }^{20,34}$ :

$$
\left[\begin{array}{l}
x \\
y
\end{array}\right]=\frac{f}{Z+l-f}\left[\begin{array}{l}
X \\
Y
\end{array}\right],
$$

where $f$, the effective focal length of the zoom lens, is related to $l$ by

$$
\frac{1}{f}=\frac{1}{f_{a}}+\frac{1}{f_{b}}-\frac{l}{f_{a} f_{b}},
$$

where $f_{a}$ and $f_{b}$ are the focal lengths of the two lens groups corresponding to the two principal planes determined during calibration. Zoom camera calibration techniques can be found in Refs. 19, 35-38. 
Table 1. Projection Models for a Zoom Camera

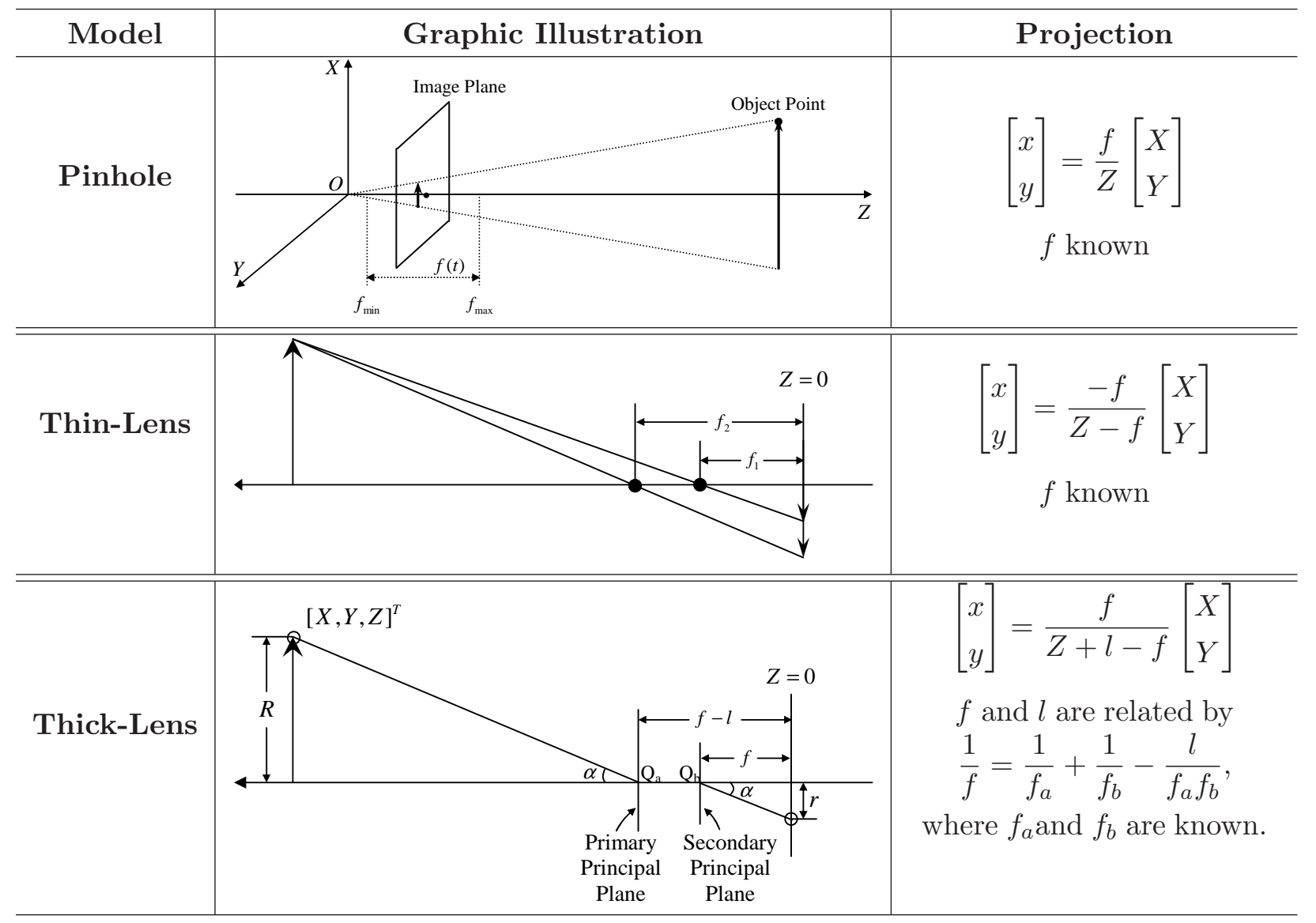

The pinhole, thin-lens and thick-lens projection models are illustrated in Table 1 .

Both thin and thick-lens models have the same capability to represent the optical properties if the lens thickness has no physical meaning ${ }^{21}$. For our purpose, the thin-lens and thick-lens models are fundamentally similar from the perspective of the mathematical derivation of the estimation problem. The thin-lens model (1) is used in the following derivation. Similar procedure can be performed for the thick-lens model. However, this is not elaborated further in this paper.

\section{Motion Estimation}

Consider the movement of the rigid object described by ${ }^{7,9}$ :

$$
\left[\begin{array}{c}
\dot{X}(t) \\
\dot{Y}(t) \\
\dot{Z}(t)
\end{array}\right]=\left[\begin{array}{ccc}
0 & \omega_{1} & \omega_{2} \\
-\omega_{1} & 0 & \omega_{3} \\
-\omega_{2} & -\omega_{3} & 0
\end{array}\right]\left[\begin{array}{c}
X(t) \\
Y(t) \\
Z(t)
\end{array}\right]+\left[\begin{array}{l}
b_{1} \\
b_{2} \\
b_{3}
\end{array}\right],
$$

where $[X(t), Y(t), Z(t)]^{\top}$ denotes the position of the feature point in the space; the matrix $\left[\omega_{i}\right]$ presents the rotational dynamics, and the vector $\left[b_{i}\right]$ corresponds to the translational motion. The observed position on the image plane of a zoom camera is defined by

$$
\left[\begin{array}{l}
x_{1}(t) \\
x_{2}(t)
\end{array}\right]=\frac{1}{Z(t)-f(t)}\left[\begin{array}{l}
X(t) \\
Y(t)
\end{array}\right]
$$


where the thin-lens model in (1) is used and $f(t)$ is the time-varying focal length.

Let

$$
\begin{aligned}
\boldsymbol{\omega} & =\left[\omega_{1}, \omega_{2}, \omega_{3}\right]^{\top}, \\
\boldsymbol{b} & =\left[b_{1}, b_{2}, b_{3}\right]^{\top} .
\end{aligned}
$$

We make the following assumptions:

1. The motion parameters $\boldsymbol{\omega}$ and $\boldsymbol{b}$ are bounded constants.

2. $Z(t)$ meets the condition $Z(t)>\epsilon>0$ for all $t \geq 0$, where $\epsilon$ is a constant.

3. $x_{1}(t)$ and $x_{2}(t)$ are bounded for all $t \geq 0$.

Our objective is to estimate $[X(t), Y(t), Z(t)]^{\top}$ and the motion parameters $\boldsymbol{\omega}$ and $\boldsymbol{b}$ by using the perspective observations of a single feature point, namely $x_{1}(t)$ and $x_{2}(t)$ as given in (5).

Let

$$
x_{3}(t)=\frac{1}{Z(t)-f(t)} .
$$

The resulting perspective dynamic system, obtained by applying equations (5) and (7) to (4), is given by:

$$
\left\{\begin{array}{l}
\dot{x}_{1}(t)=\omega_{1} x_{2}(t)+\omega_{2}\left(1+x_{1}^{2}(t)\right)+\omega_{3} x_{1}(t) x_{2}(t)+b_{1} x_{3}(t)-x_{1} b_{3} x_{3}(t)+f(t) \omega_{2} x_{3}(t)+\dot{f}(t) x_{1}(t) x_{3}(t), \\
\dot{x}_{2}(t)=-\omega_{1} x_{1}(t)+\omega_{2} x_{1}(t) x_{2}(t)+\omega_{3}\left(1+x_{2}^{2}(t)\right)+b_{2} x_{3}(t)-x_{2}(t) b_{3} x_{3}(t)+f(t) \omega_{3} x_{3}(t)+\dot{f}(t) x_{2}(t) x_{3}(t), \\
\dot{x}_{3}(t)=\left(\omega_{2} x_{1}(t)+\omega_{3} x_{2}(t)\right) x_{3}(t)-\left(b_{3}-\dot{f}(t)\right) x_{3}^{2}(t) .
\end{array}\right.
$$

It is obvious that the position of the object in the $3 \mathrm{D}$ space can be calculated as

$$
X(t)=\frac{x_{1}(t)}{x_{3}(t)}, \quad Y(t)=\frac{x_{2}(t)}{x_{3}(t)}, \quad Z(t)=\frac{1}{x_{3}(t)}+f(t),
$$

if $x_{3}(t)$ is available. We show that $\boldsymbol{\omega}, x_{3}(t)$ and $\boldsymbol{b}$ can be estimated via the following steps. Note that $f(t)$ is a design signal and is therefore available.

\section{III.A. Estimation of Rotational Parameters $\omega$}

Let

$$
\boldsymbol{\theta}(t)=\left[\begin{array}{c}
\theta_{1}(t) \\
\theta_{2}(t) \\
\theta_{3}(t) \\
\theta_{4}(t) \\
\theta_{5}(t) \\
\theta_{6}(t)
\end{array}\right] \triangleq\left[\begin{array}{c}
\left(b_{1}+f \omega_{2}\right) x_{3}(t) \\
\left(b_{2}+f \omega_{3}\right) x_{3}(t) \\
b_{3} x_{3}(t) \\
\omega_{1} \\
\omega_{2} \\
\omega_{3}
\end{array}\right] .
$$

By choosing a constant focal length such that

$$
\dot{f}(t)=0,
$$


the motion dynamics in (8) take the following form:

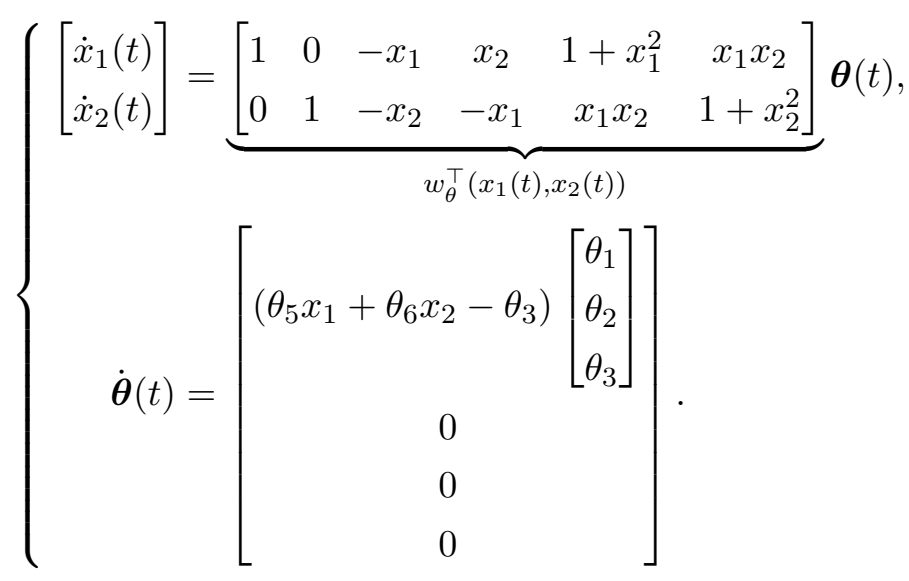

The system (11) exhibits the structure given in equation (23) in the Appendix, to which IBO may be applied by treating $\boldsymbol{x}_{1}(t)=\left[x_{1}(t), x_{2}(t)\right]^{\top}, \boldsymbol{x}_{2}(t)=\boldsymbol{\theta}(t)$, and $\phi\left(\boldsymbol{x}_{1}, \boldsymbol{u}\right)=0$. The IBO is briefly reviewed in the Appendix. To apply the IBO, we need the following assumption for the system (11):

Assumption III.1 There do not exist constants $\kappa_{i}($ for $i=1, \ldots, 6)$ with $\sum_{i=1}^{6} \kappa_{i}^{2} \neq 0$ such that

$$
\sum_{i=1}^{6} \kappa_{i} v_{i}(\tau)=0
$$

for all $\tau \in[t, t+\mu]$, where $v_{i}(\tau)$ stands for the $i^{\text {th }}$ column in $w_{\theta}^{\top}\left(x_{1}(t), x_{2}(t)\right)$ in (11) and $\mu$ is a small positive constant.

Let $\hat{\boldsymbol{\theta}}(t)$ denote the estimate of $\boldsymbol{\theta}(t)$. The following observer can be designed for the system in (11):

$$
\left\{\begin{aligned}
{\left[\begin{array}{c}
\dot{\hat{x}}_{1}(t) \\
\dot{\hat{x}}_{2}(t)
\end{array}\right] } & =G A\left[\begin{array}{l}
\hat{x}_{1}(t)-x_{1}(t) \\
\hat{x}_{2}(t)-x_{2}(t)
\end{array}\right]+w_{\theta}^{\top}\left(x_{1}(t), x_{2}(t)\right) \hat{\boldsymbol{\theta}}(t), \\
\dot{\hat{\boldsymbol{\theta}}}(t) & =-G^{2} w_{\theta}\left(x_{1}(t), x_{2}(t)\right) P\left[\begin{array}{l}
\hat{x}_{1}(t)-x_{1}(t) \\
\hat{x}_{2}(t)-x_{2}(t)
\end{array}\right]+\left[\begin{array}{c}
\left(\hat{\theta}_{5} x_{1}+\hat{\theta}_{6} x_{2}-\hat{\theta}_{3}\right)\left[\begin{array}{c}
\hat{\theta}_{1} \\
\hat{\theta}_{2} \\
\hat{\theta}_{3}
\end{array}\right] \\
0 \\
0
\end{array}\right] .
\end{aligned}\right.
$$

According to Theorem V.1, there exists a positive constant $G_{0}$ such that choosing $G>G_{0}$ ensures that the estimation errors

$$
\left[\hat{x}_{1}(t)-x_{1}(t), \hat{x}_{2}(t)-x_{2}(t), \hat{\boldsymbol{\theta}}(t)^{\top}-\boldsymbol{\theta}^{\top}\right]^{\top}
$$

converge to zero exponentially. This gives $\hat{\boldsymbol{\omega}}(t)$. Clearly,

$$
\hat{\boldsymbol{\omega}}(t)=\left[\hat{\theta}_{4}(t), \hat{\theta}_{5}(t), \hat{\theta}_{6}(t)\right]^{\top},
$$


where $\hat{\theta}_{i}(t)$ (for $\left.i=4,5,6\right)$ denotes the $i^{\text {th }}$ element of $\hat{\boldsymbol{\theta}}(t)$. The signals $\hat{\boldsymbol{\omega}}(t)$ will be used later to estimate $x_{3}(t)$ and $\boldsymbol{b}$.

The IBO has been applied to the range identification problem assuming some or all of the motion parameters are known ${ }^{7,39}$. In this paper, all the motion parameters are assumed to be unknown constants. The objective is to estimate the position and the motion parameters via observations of a single feature point using a zoom camera. The IBO's exponential convergence property makes this approach viable.

\section{III.B. Estimation of Unknown Depth $x_{3}(t)$}

Let

$$
\boldsymbol{\vartheta}(t)=\left[\begin{array}{c}
\vartheta_{1}(t) \\
\vartheta_{2}(t) \\
\vartheta_{3}(t) \\
\vartheta_{4}(t)
\end{array}\right] \triangleq\left[\begin{array}{c}
b_{1} x_{3}(t) \\
b_{2} x_{3}(t) \\
b_{3} x_{3}(t) \\
x_{3}(t)
\end{array}\right] .
$$

The system dynamics in (8) can be rewritten as

$$
\left\{\begin{aligned}
{\left[\begin{array}{c}
\dot{x}_{1}(t) \\
\dot{x}_{2}(t)
\end{array}\right] } & =\left[\begin{array}{c}
\omega_{1} x_{2}+\omega_{2}\left(1+x_{1}^{2}\right)+\omega_{3} x_{1} x_{2} \\
-\omega_{1} x_{1}+\omega_{2} x_{1} x_{2}+\omega_{3}\left(1+x_{2}^{2}\right)
\end{array}\right]+\underbrace{\left[\begin{array}{cccc}
1 & 0 & -x_{1} & f(t) \omega_{2}+\dot{f}(t) x_{1} \\
0 & 1 & -x_{2} & f(t) \omega_{3}+\dot{f}(t) x_{2}
\end{array}\right]}_{w_{\vartheta}^{\top}\left(x_{1}(t), x_{2}(t)\right)} \boldsymbol{\vartheta}(t), \\
\dot{\boldsymbol{\vartheta}}(t) & =\left(\omega_{2} x_{1}+\omega_{3} x_{2}-\vartheta_{3}+\dot{f}(t) \vartheta_{4}\right) \boldsymbol{\vartheta}(t) .
\end{aligned}\right.
$$

Replacing $\boldsymbol{\omega}=\left[\omega_{1}, \omega_{2}, \omega_{3}\right]^{\top}$ by $\hat{\boldsymbol{\omega}}(t)$, where $\hat{\boldsymbol{\omega}}(t)$ is obtained in (14), the system (16) also fits into the form to which IBO can be applied. Accordingly, an observer for $\boldsymbol{\vartheta}(t)$ can be constructed. Similarly, we need the following assumption:

Assumption III.2 There do not exist constants $\kappa_{i}($ for $i=1, \ldots, 4)$ with $\sum_{i=1}^{4} \kappa_{i}^{2} \neq 0$ such that

$$
\sum_{i=1}^{4} \kappa_{i} v_{i}(\tau)=0,
$$

for all $\tau \in[t, t+\mu]$, where $v_{i}(\tau)$ stands for the $i^{\text {th }}$ column in $w_{\vartheta}^{\top}\left(x_{1}(t), x_{2}(t)\right)$ in (16) and $\mu$ is a small positive constant.

To achieve the observability condition in (17), the camera's focal length is designed to perform the following sinusoidal motion:

$$
f(t)=0.5 \sin (2 \pi t) .
$$

Detailed observer for the system in (16) is not given here (see Sec. III.A for a similar procedure). Let $\hat{\boldsymbol{\vartheta}}(t)$ denote the estimate of $\boldsymbol{\vartheta}(t)$. Clearly,

$$
\hat{x}_{3}(t)=\hat{\vartheta}_{4}(t)
$$

where $\hat{\vartheta}_{4}(t)$ is the $4^{\text {th }}$ element of $\hat{\boldsymbol{\vartheta}}(t)$. The signal $\hat{x}_{3}(t)$, together with $\hat{\boldsymbol{\omega}}(t)$ that has been estimated in Sec. III.A, will be used later to estimate the translational parameters $\boldsymbol{b}$. Note that upon obtaining $\hat{x}_{3}(t)$, estimate of the $3 \mathrm{D}$ position $[X(t), Y(t), Z(t)]^{\top}$ can be computed directly from $\hat{x}_{3}(t)$ and the visual measurements $x_{1}(t)$ and $x_{2}(t)$. 


\section{III.C. Estimation of Translational Parameters $b$}

From Sec. III.B, the translational parameters $\boldsymbol{b}$ can be directly calculated by using the estimates of $\left(\boldsymbol{b}(t) x_{3}(t)\right)$ and $x_{3}(t)$. However, this approach is not preferable when $x_{3}(t)$ is small and estimation errors are not negligible ${ }^{12,40}$. Alternately, we can construct a dynamic observer for the parameters $\boldsymbol{b}$. Under the assumption that $\boldsymbol{b}$ are constants, the first two equations in (8) can be rewritten to give the following equations in the form of equation (23):

$$
\left\{\begin{aligned}
{\left[\begin{array}{c}
\dot{x}_{1}(t) \\
\dot{x}_{2}(t)
\end{array}\right] } & =\left[\begin{array}{c}
\omega_{1} x_{2}+\omega_{2}\left(1+x_{1}^{2}\right)+\omega_{3} x_{1} x_{2} \\
-\omega_{1} x_{1}+\omega_{2} x_{1} x_{2}+\omega_{3}\left(1+x_{2}^{2}\right)
\end{array}\right]+\left[\begin{array}{c}
f(t) \omega_{2}+\dot{f}(t) x_{1} \\
f(t) \omega_{3}+\dot{f}(t) x_{2}
\end{array}\right] x_{3}+\left[\begin{array}{ccc}
1 & 0 & -x_{1} \\
0 & 1 & -x_{2}
\end{array}\right] x_{3} \boldsymbol{b}, \\
\dot{\boldsymbol{b}} & =0
\end{aligned}\right.
$$

Replacing $\boldsymbol{\omega}=\left[\omega_{1}, \omega_{2}, \omega_{3}\right]^{\top}$ by $\hat{\boldsymbol{\omega}}(t)$ and $x_{3}(t)$ by $\hat{x}_{3}(t)$, where $\hat{\boldsymbol{\omega}}(t)$ and $\hat{x}_{3}(t)$ are obtained in (14) and (19), respectively, an observer for $\boldsymbol{b}$ can be constructed by applying IBO to (20). Note that if Assumption III.2 holds, the convergence condition for the observer of (20) is also satisfied.

In summary, complete 3D estimation of a rigid-body motion is given in equations (13), (19) and by constructing an observer to (20). Our proposed method is feasible due to the following factors:

1. The assumption that the motion parameters are unknown constants allows for transforming the resulting perspective dynamic system into a linearly parameterized structure, such that existing perspective nonlinear observers can be applied (see equations (11), (16) and (20)).

2. The depth ambiguity is resolved applying adjustable focal length.

\section{Simulation Results}

Considering the system in (8), motion estimation of a rigid-body with constant rotational and translational parameters is performed for the following dynamical system:

$$
\begin{aligned}
& {\left[\begin{array}{c}
\dot{X}(t) \\
\dot{Y}(t) \\
\dot{Z}(t)
\end{array}\right]=\left[\begin{array}{ccc}
0 & 5 & 0.6 \\
-5 & 0 & 0.7 \\
-0.6 & -0.7 & 0
\end{array}\right]\left[\begin{array}{c}
X(t) \\
Y(t) \\
Z(t)
\end{array}\right]+\left[\begin{array}{c}
3 \\
2 \\
-0.1
\end{array}\right],} \\
& \left(X_{0}, Y_{0}, Z_{0}\right)=(1,1.5,2.5), \quad x_{0}=\left(X_{0}, Y_{0}, Z_{0}\right) / Z_{0} .
\end{aligned}
$$

In simulations, the visual measurements are corrupted with $1 \%$ uniform noise and a step size of 0.02 second is used. The observers' initial states are chosen to be $\hat{\boldsymbol{\theta}}(0)=\boldsymbol{\theta}(0)+[0.8,-0.5,0.7,0.5,0.5,0.5]^{\top}$, $\hat{\boldsymbol{\vartheta}}(0)=\boldsymbol{\vartheta}(0)+[0.8,-0.8,0.5,1]^{\top}$, and $\hat{\boldsymbol{b}}(0)=[4,3,0.9]^{\top}$. The observer parameters are chosen to be $M=15, A=\mathbb{I}_{2}, P=-1 / 2 \times \mathbb{I}_{2}$, and $G=5$, where $\mathbb{I}_{2}$ denotes the $2 \times 2$ identity matrix. Without loss of generality, the camera's focal length $f$ is fixed at 0.5 when constructing the observer (13). Later on, the focal length employs the sinusoidal motion in (18).

Estimations of $\boldsymbol{\omega}, x_{3}(t)$ and $\boldsymbol{b}$ are shown in Figures 1, 2, and 3, respectively, where true values are plotted in solid lines and corresponding estimates are in dashed. It can be seen that good estimates are obtained. Figure 4 shows the advantage of using the nonlinear observer to estimate $\boldsymbol{b}$ using direct computations by plotting the errors of these two methods for comparison. 

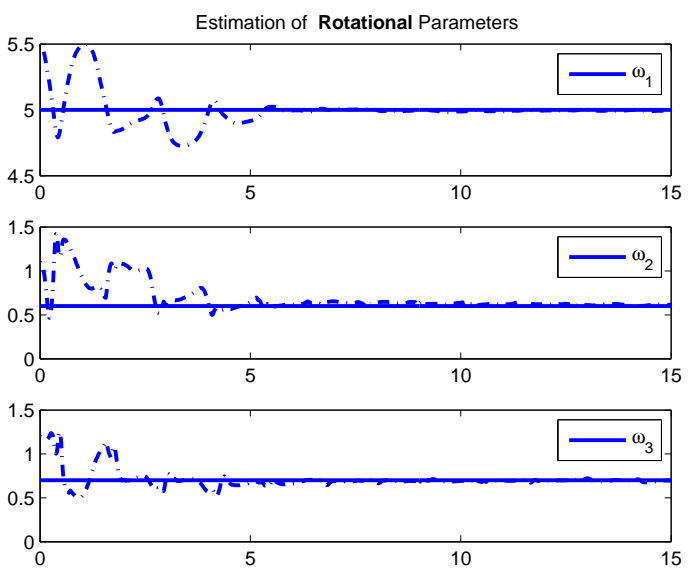

Figure 1. Estimation of rotational parameters $\omega$ of (21).

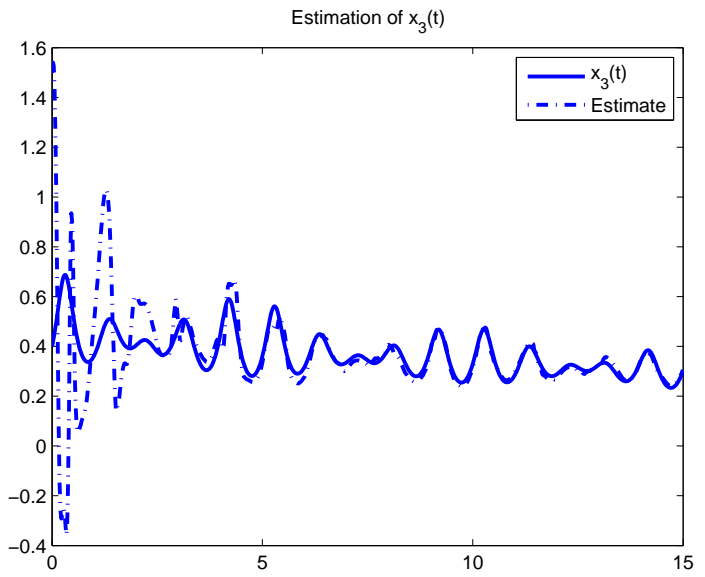

Figure 2. Estimation of $x_{3}(t)$ of (21).

Figures 5.7 show simulation results of the following motion dynamics

$$
\begin{aligned}
& {\left[\begin{array}{c}
\dot{X}(t) \\
\dot{Y}(t) \\
\dot{Z}(t)
\end{array}\right]=\left[\begin{array}{ccc}
0 & 4 & 0.5 \\
-4 & 0 & 0.7 \\
-0.5 & -0.7 & 0
\end{array}\right]\left[\begin{array}{c}
X(t) \\
Y(t) \\
Z(t)
\end{array}\right]+\left[\begin{array}{c}
4 \\
3 \\
-0.2
\end{array}\right],} \\
& \left(X_{0}, Y_{0}, Z_{0}\right)=(1,1.5,2.5), \quad x_{0}=\left(X_{0}, Y_{0}, Z_{0}\right) / Z_{0} .
\end{aligned}
$$

Similar to the simulations conducted on (21), the visual measurements are corrupted with $1 \%$ uniform noise and a step size of 0.02 second is used. The observers' initial states are chosen to be $\hat{\boldsymbol{\theta}}(0)=\boldsymbol{\theta}(0)+[0.8,-0.5,0.7,0.5,0.5,0.5]^{\top}, \hat{\boldsymbol{\vartheta}}(0)=\boldsymbol{\vartheta}(0)+[0.8,-0.8,0.5,1]^{\top}$, and $\hat{\boldsymbol{b}}(0)=[5,4,0.8]^{\top}$. It can be observed that both position estimation of $x_{3}(t)$ and parameter estimations of $\boldsymbol{\omega}$ and $\boldsymbol{b}$ are obtained.

9 

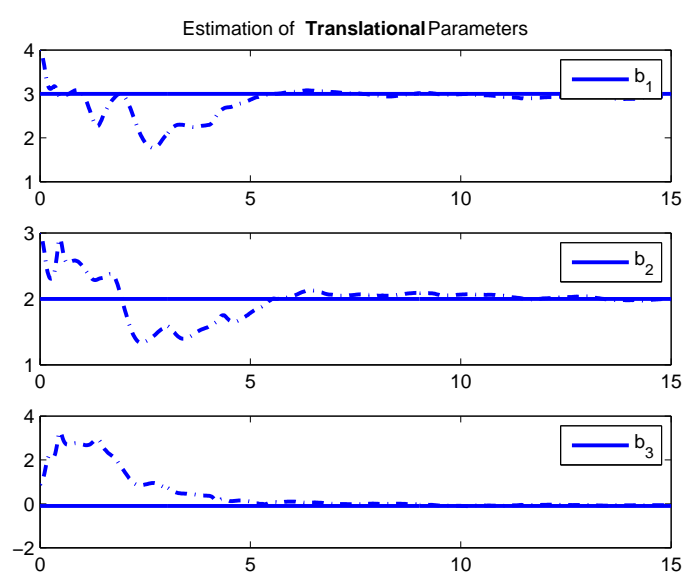

Figure 3. Estimation of translational parameters $b$ of (21).
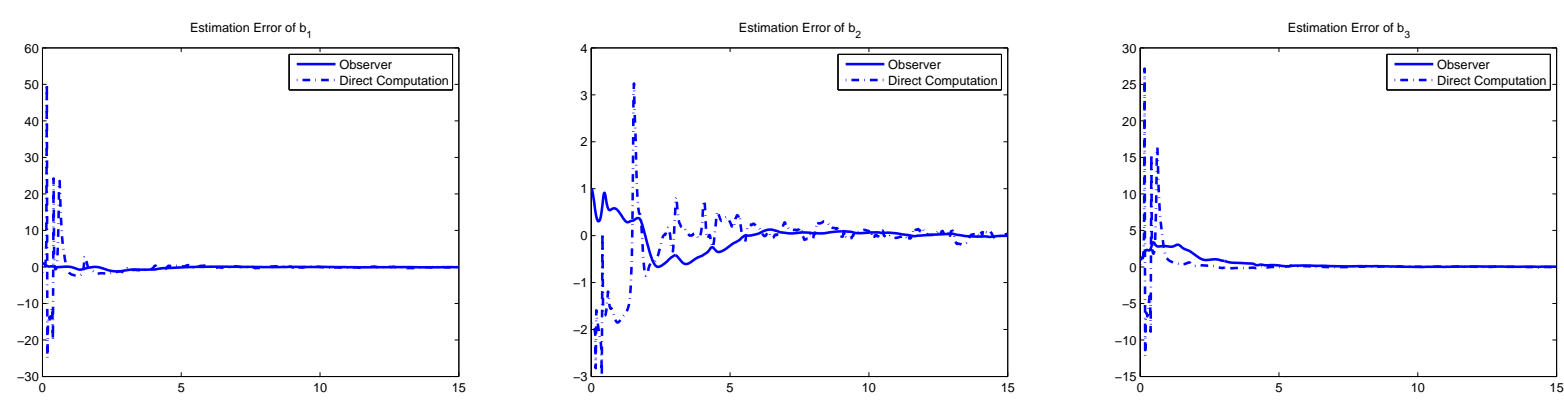

Figure 4. Comparison of estimation errors of the translational parameters between observer outputs (solid lines) and direct computations (dashed lines) of (21).

\section{Conclusion}

We present a solution to the estimation problem of a rigid-body motion using a zoom camera via observations of a single feature point. Assuming that all the motion parameters are unknown constants, these parameters, together with the unknown depth/range, can be estimated via two steps. In the first step, the zoom camera is used as a fixed focal-length camera. Similar to results available in the literature, the rotational parameters of the rigid-body motion can be estimated. The second step relies on the camera's adjustable focal length, which is required to perform a time-varying motion to provide persistent excitation for the estimation of the unknown range along with the translational parameters. A sinusoidal motion is currently adopted in this step.

By exploiting a unique intrinsic parameter to the active vision system, i.e., the adjustable focal length (also known as zoom), motion estimation of a rigid-body motion can be achieved without additional information or extra sensors. Having the estimation achieved in one unit (i.e., the zoom camera), the vehicle's behavior need not be modified. The image processing task can perceivably be simplified as well, since the feature point correspondence problem is avoided. 

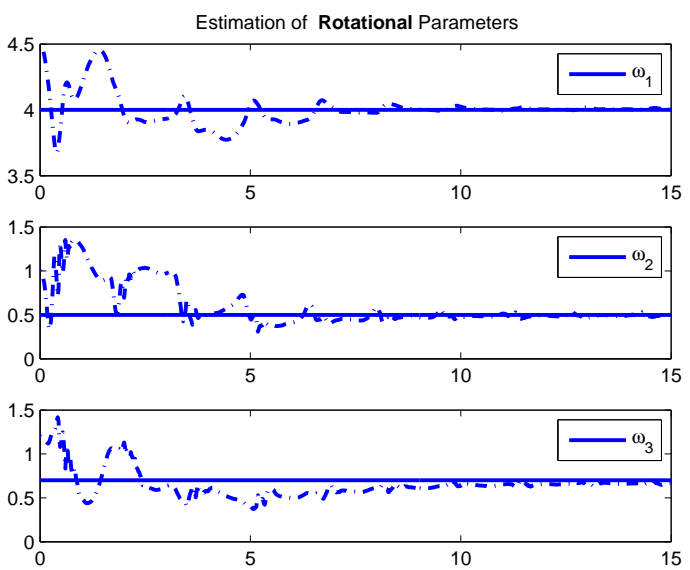

Figure 5. Estimation of rotational parameters $\omega$ of (22).

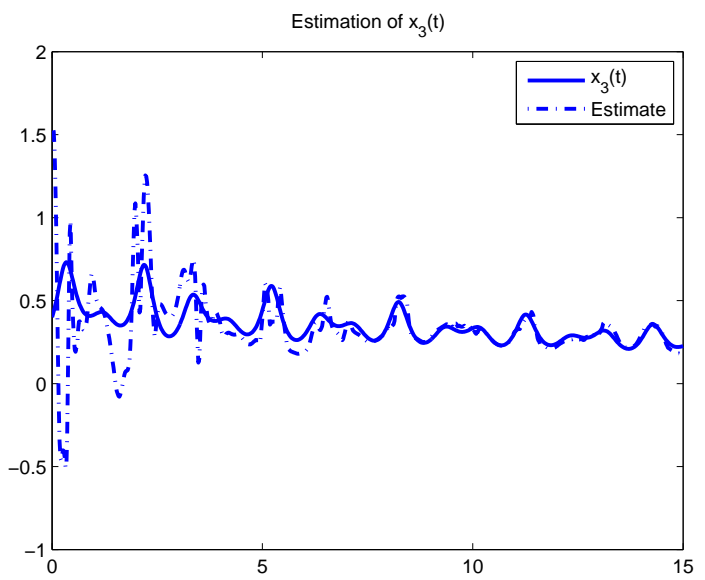

Figure 6. Estimation of $x_{3}(t)$ of (22).

\section{Acknowledgments}

This work was sponsored in part by ARO Grant \#W911NF-06-1-0330 and AFOSR MURI subcontract F49620-03-1-0401.

\section{Review of IBO}

We review the IBO in this section. This observer is used for the estimation task. The convergence properties of IBO are proved for a class of nonlinear systems with the following structure ${ }^{7}$ :

$$
\left\{\begin{aligned}
\dot{\boldsymbol{x}}_{1}(t) & =w^{\top}\left(\boldsymbol{x}_{1}(t), \boldsymbol{u}(t)\right) \boldsymbol{x}_{2}(t)+\phi\left(\boldsymbol{x}_{1}(t), \boldsymbol{u}(t)\right), \\
\dot{\boldsymbol{x}}_{2}(t) & =g\left(\boldsymbol{x}_{1}(t), \boldsymbol{x}_{2}(t), \boldsymbol{u}(t)\right), \\
\boldsymbol{y}(t) & =\boldsymbol{x}_{1}(t),
\end{aligned}\right.
$$

where $\boldsymbol{x}_{1}(t) \in \mathbb{X}_{1} \subset \mathbb{R}^{n_{1}}, \boldsymbol{x}_{2}(t) \in \mathbb{X}_{2} \subset \mathbb{R}^{n_{2}}$, and $\boldsymbol{u}(t) \in \mathbb{U} \subset \mathbb{R}^{k}$. The $n_{1} \times n_{2}$ matrix $w^{\top}\left(\boldsymbol{x}_{1}(t), \boldsymbol{u}(t)\right)$ and the vector $g\left(\boldsymbol{x}_{1}(t), \boldsymbol{x}_{2}(t), \boldsymbol{u}(t)\right)$ are general nonlinear functions of their parameters. Let $\boldsymbol{x}(t)=$ 

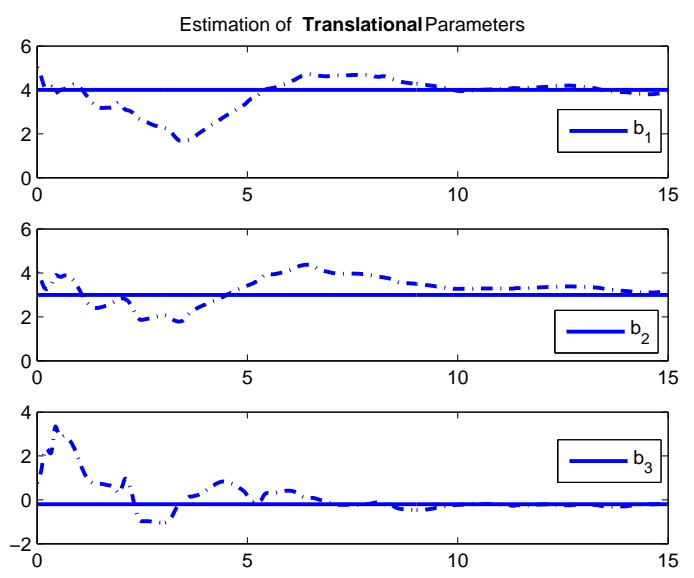

Figure 7. Estimation of translational parameters $b$ of (22).

$\left[\boldsymbol{x}_{1}^{\top}(t), \boldsymbol{x}_{2}^{\top}(t)\right]^{\top}$ and $n=n_{1}+n_{2}$, so that $\boldsymbol{x}(t) \in \mathbb{X} \subset \mathbb{R}^{n}$, where $\mathbb{X}=\mathbb{X}_{1} \oplus \mathbb{X}_{2}$.

Following Ref. 7, we introduce the following assumptions:

\section{Assumption V.1}

1. Let $\boldsymbol{x}(t)$ be bounded: $\|\boldsymbol{x}(t)\|<M$, where $M>0$ for every $t \geq 0$. Let $\Omega=\left\{\boldsymbol{x} \in \mathbb{R}^{n}:\|\boldsymbol{x}(t)\|<\right.$ $M\}$. Further, for some fixed constant $\gamma>1$, let $\Omega_{\gamma}=\left\{\boldsymbol{x}(t) \in \mathbb{R}^{n}:\|\boldsymbol{x}(t)\|<\gamma M\right\}$. Assume that the function $g\left(\boldsymbol{x}_{1}(t), \boldsymbol{x}_{2}(t), \boldsymbol{u}(t)\right)$ is locally Lipschitz in $\Omega_{\gamma}$ with respect to $\boldsymbol{x}_{2}(t)$, i.e., there exists a positive constant $\alpha$ such that

$$
\left\|g\left(\boldsymbol{x}_{1}, \boldsymbol{x}_{2}, \boldsymbol{u}\right)-g\left(\boldsymbol{x}_{1}, \boldsymbol{z}_{2}, \boldsymbol{u}\right)\right\|<\alpha\left\|\boldsymbol{x}_{2}-\boldsymbol{z}_{2}\right\|,
$$

for all $\boldsymbol{x}_{2}(t), \boldsymbol{z}_{2}(t) \in \Omega_{\gamma} \cap \mathbb{X}_{2}$, uniformly in $\boldsymbol{x}_{1}(t) \in \Omega_{\gamma} \cap \mathbb{X}_{1}$ and $\boldsymbol{u}(t) \in \mathbb{U}$.

2. Let the regressor matrix $w^{\top}\left(\boldsymbol{x}_{1}(t), \boldsymbol{u}(t)\right)$ and its first time derivative be piecewise smooth and uniformly bounded. Further, assume that there exist positive constants $L_{1}, L_{2}, \eta$, and $\mu$ such that

$$
\begin{gathered}
\left\|w^{\top}\left(\boldsymbol{x}_{1}, \boldsymbol{u}\right)\right\|<L_{1}, \quad\left\|\frac{\mathrm{d} w^{\top}\left(\boldsymbol{x}_{1}, \boldsymbol{u}\right)}{\mathrm{dt}}\right\|<L_{2}, \\
\int_{t}^{t+\mu} w\left(\boldsymbol{x}_{1}(\tau), \boldsymbol{u}(\tau)\right) w^{\top}\left(\boldsymbol{x}_{1}(\tau), \boldsymbol{u}(\tau)\right) \mathrm{d} \tau>\eta \mathbb{I}
\end{gathered}
$$

for all $t \geq 0$, for all trajectories that originate in $\mathbb{X}$ and for all $\boldsymbol{u}(t) \in \mathbb{U}$, where $\mathbb{I}$ denotes the identity matrix of appropriate dimension.

Letting $\hat{\boldsymbol{x}}_{1}(t)$ and $\hat{\boldsymbol{x}}_{2}(t)$ be the estimates of $\boldsymbol{x}_{1}(t)$ and $\boldsymbol{x}_{2}(t)$, respectively, the IBO is introduced as:

$$
\left\{\begin{array}{l}
\dot{\hat{\boldsymbol{x}}}_{1}(t)=G A\left(\hat{\boldsymbol{x}}_{1}-\boldsymbol{x}_{1}\right)+w^{\top}\left(\boldsymbol{x}_{1}, \boldsymbol{u}\right) \hat{\boldsymbol{x}}_{2}+\phi\left(\boldsymbol{x}_{1}, \boldsymbol{u}\right), \\
\dot{\hat{\boldsymbol{x}}}_{2}(t)=-G^{2} w\left(\boldsymbol{x}_{1}, \boldsymbol{u}\right) P\left(\hat{\boldsymbol{x}}_{1}-\boldsymbol{x}_{1}\right)+g\left(\boldsymbol{x}_{1}, \hat{\boldsymbol{x}}_{2}, \boldsymbol{u}\right), \\
\hat{\boldsymbol{x}}\left(t_{i}^{+}\right)=M \frac{\hat{\boldsymbol{x}}\left(t_{i}^{-}\right)}{\left\|\hat{\boldsymbol{x}}\left(t_{i}^{-}\right)\right\|},
\end{array}\right.
$$


where $G$ is a scalar constant and $A$ is an $n_{1} \times n_{1}$ Hurwitz matrix. The matrix $P$ is the positive definite solution of the Lyapunov equation $A^{\top} P+P A=-Q$ for some $Q>0$. The sequence $t_{i}$ is defined as follows:

$$
t_{i}=\min \left\{t: t>t_{i-1} \text { and }\|\hat{\boldsymbol{x}}(t)\| \geq \gamma M\right\}, t_{0}=0,
$$

where $\gamma$ is a fixed constant.

Theorem V.1 ${ }^{7}$ Subject to Assumption V.1, there exists a positive constant $G_{0}$ such that the estimation errors $\boldsymbol{e}_{1}(t)=\boldsymbol{x}_{1}(t)-\hat{\boldsymbol{x}}_{1}(t)$ and $\boldsymbol{e}_{2}(t)=\boldsymbol{x}_{2}(t)-\hat{\boldsymbol{x}}_{2}(t)$ converge to zero exponentially if the constant $G$ in (26) is chosen larger than $G_{0}$.

\section{References}

${ }^{1}$ Cho, H., Lee, K., and Lee, S., "A New Robust 3D Motion Estimation Under Perspective Projection," IEEE Conference on Image Processing, Thessaloniki, Greece, Oct. 2001, pp. 660-663.

${ }^{2}$ Diamantaras, K., Papadimitrion, T., Strintzis, M., and Roumeliotis, M., "Total Least Squares 3-D Motion Estimation," IEEE Conference on Image Processing, Chicago, IL, Oct. 1998, pp. 923-927.

${ }^{3}$ Diamantaras, K. and Strintzis, M., "Camera Motion Parameter Recovery Under Perspective Projection," IEEE Conference on Image Processing, Switzerland, Sep. 1996, pp. 807-810.

${ }^{4}$ Papadimitriou, T., Diamantaras, K., Strintzis, M., and Roumeliotis, M., "Robust Estimation of Rigid-Body 3-D Motion Parameters Based on Point Correspondences," IEEE Transactions on Circuits and Systems for Video Technology, Vol. 10, No. 4, June 2000, pp. 541-549.

${ }^{5}$ Chiuso, A., Favaro, P., Jin, H., and Soatto, S., "Structure from Motion Causally Integrated Over Time," IEEE Transactions on Pattern Analysis and Machine Intelligence, Vol. 24, No. 4, Apr. 2002, pp. 523-535.

${ }^{6}$ Soatto, S., Frezza, R., and Perona, P., "Motion Estimation via Dynamic Vision," IEEE Transactions on Automatic Control, Vol. 41, No. 3, Dec. 1996, pp. 393-413.

${ }^{7}$ Jankovic, M. and Ghosh, B., "Visually Guided Ranging from Observations of Points, Lines and Curves via an Identifier Based nonlinear Observer," Systems and Control Letters, Vol. 25, 1995, pp. 63-73.

${ }^{8}$ Chen, X. and Kano, H., "A New State Observer for Perspective Systems," IEEE Transactions on Automatic Control, Vol. 47, No. 4, Apr. 2002, pp. 658-663.

${ }^{9}$ Dixon, W., Fang, Y., Dawson, D., and Flynn, T., "Range Identification for Perspective Vision Systems," IEEE Transactions on Automatic Control, Vol. 48, No. 12, Dec. 2003, pp. 2232-2238.

${ }^{10} \mathrm{Ma}$, L., Chen, Y., and Moore, K., "Range Identification for Perspective Dynamic Systems Using Linear Approximation," IEEE Conference on Robotics and Automation, New Orleans, Apr.-May 2004, pp. 1658-1663.

${ }^{11}$ Karagiannis, D. and Astolfi, A., "A New Solution to the Problem of Range Identification in Perspective Vision Systems," IEEE Transactions on Automatic Control, Vol. 50, No. 12, Dec. 2005, pp. 2074-2077.

${ }^{12}$ Chen, X. and Kano, H., "State Observer for a Class of Nonlinear Systems and Its Application to Machine Vision," IEEE Transactions on Automatic Control, Vol. 49, No. 11, Nov. 2004, pp. 2085-2091.

${ }^{13}$ Ghosh, B. and Martin, C., "Homogeneous Dynamical Systems Theory," IEEE Transactions on Automatic Control, Vol. 47, No. 3, Mar. 2002, pp. 462-472.

${ }^{14}$ Takahashi, S. and Ghosh, B., "Motion and Shape Identification with Vision and Range," IEEE Transactions on Automatic Control, Vol. 47, No. 8, Aug. 2002, pp. 1392-1396.

${ }^{15}$ Cao, C., Hovakimyan, N., and Evers, J., "Active Control of Visual Sensor for Aerial Tracking," Proceedings of AIAA Guidance, Navigation and Control Conference, Keystone, CO, Aug. 2006, AIAA-2006-6610.

${ }^{16}$ Cao, C. and Hovakimyan, N., "Vision-Based Tracking using Intelligent Excitation," International Journal of Control, 2008, To Appear.

${ }^{17} \mathrm{Ma}$, J. and Olsen, S., "Depth From Zooming," Journal of the Optical Society of America. A, Optics and image science, Vol. 7, No. 10, Oct. 1990, pp. 1883-1890.

${ }^{18}$ Lavest, J., Rives, G., and Dhome, M., "Three-Dimensional Reconstruction by Zooming," IEEE Transactions on Robotics and Automation, Vol. 9, No. 2, Apr. 1993, pp. 196-207. 
${ }^{19}$ Lavest, J., Delherm, C., P.euchot, B., and Daucher, N., "Implicit Reconstruction by Zooming," Computer Vision and Image Understanding, Vol. 66, No. 3, June 1997, pp. 301-315.

${ }^{20}$ Fayman, J., Sundarsky, O., Rivlin, E., and Rudzsky, M., "Zoom Tracking and Its Applications," Machine Vision and Applications, Vol. 13, No. 1, Aug. 2001, pp. 25-37.

${ }^{21}$ Baba, M., Asada, N., Oda, A., and Migita, T., "A Thin Lens Based Camera Model for Depth Estimation from Defocus and Translation by Zooming," International Conference on Vision Interface, Calgary, May 2002, pp. $274-281$.

${ }^{22}$ Alenya, G., Alberich, M., and Torras, C., "Depth from the Visual Motion of a Planar Target Induced by Zooming," IEEE Conference on Robotics and Automation, Apr. 2007, pp. 4727-4732.

${ }^{23}$ Ma, L., Chen, Y., and Moore, K., "Range Identification for Perspective Dynamic System with a Single Homogeneous Observation," International Journal of Applied Mathematics and Computer Science, Vol. 15, No. 1, 2005, pp. 63-72.

${ }^{24}$ Dahl, O., Nyberg, F., Holst, J., and Heyden, A., "Linear Design of a Nonlinear Observer for Perspective Systems," IEEE Conference on Robotics and Automation, Barcelona, Spain, Apr. 2005, pp. 429-435.

${ }^{25}$ Ma, L., Chen, Y., and Moore, K., "Perspective Dynamic Systems with 3D Imaging Surfaces," American Control Conference, Portland, OR, June 2005, pp. 3671-3675.

${ }^{26}$ Gupta, S., Aiken, D., Hu, G., and Dixon, W., "Lyapunov-Based Range and Motion Identification for A Nonaffine Perspective Dynamic System," American Control Conference, Minneapolis, MN, June 2006, pp. 44714476.

${ }^{27}$ Aiken, D., Gupta, S., Hu, G., and Dixon, W., "Lyapunov-Based Range Identification for A Paracatadioptric System," IEEE Conference on Decision and Control, San Diego, CA, Dec. 2006, pp. 3879-3884.

${ }^{28}$ Ghosh, B., Loucks, E., and Jankovic, M., "An Introduction to Perspective Observability and Recursive Identification Problems in Machine Vision," IEEE Conference on Decision and Control, 1994, pp. 3229-3234.

${ }^{29}$ Ghosh, B., Jankovic, M., and Loucks, E., "On the Problem of Observing Motion and Shape," IEEE Conference on Automation, Robotics, and Computer Vision, Singapore, Nov. 1994, pp. 653-657.

${ }^{30}$ Dobrokhodov, V., Kaminer, I., Jones, K., Kitsios, I., Cao, C., Ma, L., Hovakimyan, N., and Woolsey, C., "Rapid Motion Estimation of a Target Moving with Time-Varying Velocity," Proceedings of AIAA Guidance, Navigation and Control Conference, Hilton Head, SC, Aug. 2007, AIAA-2007-6746.

${ }^{31} \mathrm{Ma}$, L., Cao, C., Hovakimyan, N., Woolsey, C., Dobrokhodov, V., and Kaminer, I., "Development of a VisionBased Guidance Law for Tracking a Moving Target," Proceedings of AIAA Guidance, Navigation and Control Conference, Hilton Head, SC, Aug. 2007, AIAA-2007-6744.

${ }^{32}$ Hespanha, J., Yakimenko, O., Kaminer, I., and Pascoal, A., "Linear Parametrically Varying Systems with Brief Instabilities: An Application to Integrated Vision/IMU Navigation," IEEE Transactions on Aerospace and Electronics, Vol. 40, No. 4, July 2004, pp. 889-902.

${ }^{33}$ Pascoal, A. and Oliveira, I. K. P., "Navigation System Design Using Time-Varying Complementary Filters," IEEE Transactions on Aerospace and Electronic Systems, Vol. 36, No. 4, Oct. 2000, pp. 1099-1114.

${ }^{34}$ Subbarao, M., "Parallel Depth Recovery by Changing Camera Parameters," Radiometry, 1992, pp. 340-346.

${ }^{35}$ Willson, R., Modeling and Calibration of Automated Zoom Lenses, Ph.D. thesis, Carnegie Mellon University, Pittsburgh, PA, USA, 1994.

${ }^{36} \mathrm{Li}$, M. and Lavest, J., "Some Aspects of Zoom Lens Camera Calibration," IEEE Transactions on Pattern Analysis and Machine Intelligence, Vol. 18, No. 11, Nov. 1996, pp. 1105-1110.

${ }^{37}$ Malis, E. and Cipolla, R., "Self-Calibration of Zooming Cameras Observing an Unknown Planar Structure," IEEE Conference on Pattern Recognition, Sep. 2000, pp. 85-88.

${ }^{38}$ Chen, I. and Wang, S., "An Efficient Approach for the Calibration of Multiple PTZ Cameras," IEEE Transactions on Automation Science and Engineering, Vol. 4, No. 2, Apr. 2007, pp. 286-293.

${ }^{39} \mathrm{Ma}$, L., Cao, C., Hovakimyan, N., Dixon, W., and Woolsey, C., "Range Identification in the Presence of Unknown Motion Parameters for Perspective Vision Systems," American Control Conference, New York, NY, July 2007, pp. 972-977.

${ }^{40}$ Wang, J., Ahuja, N., and Huang, T., "Optimal Motion and Structure Estimation," IEEE Transactions on Pattern Analysis and Machine Intelligence, Vol. 15, No. 9, Sep. 1993, pp. 864-884. 